\title{
Prevalence of hookworm infection and associated factors among pregnant women attending antenatal care at governmental health centers in DEMBECHA district, north West Ethiopia, 2017
}

Sewnet Wongiel Misikir ${ }^{1 *}$, Moges Wobie ${ }^{2}$, Mengistie Kassahun Tariku ${ }^{3}$ and Simachew Animen Bante ${ }^{4}$

\begin{abstract}
Background: Hookworm infection is a major public health problem in developing countries. The main way people become infected with hookworm's larva is through direct skin contact with contaminated soil when walking on barefoot. It is one of a major cause of anemia in pregnant women. The objective was to assess the prevalence and associated factors of hookworm infection among pregnant women who attended antenatal care at governmental health centers in Dembecha district, Ethiopia, 2017.

Methods: Institutional based cross sectional study was conducted on 306 pregnant women. Study participants were selected by systematic random sampling technique from February 1 to March 30, 2017. Data collectors and supervisors were trained. Semi-structured Amharic version questionnaire was used to collect data using face to face interview technique and stool sample was examined. Collected data were entered by using Epi data version 3.1 and exported to SPSS. The exported data was analyzed and presented by using descriptive summary statistics and tables. After bivariate logistic regression analysis, all variables with a $p$-value $<0.25$ were entered into multivariate logistic regression and $p$ value $<0.05$ considered as significantly associated with the outcome variable.

Results: Prevalence of hookworm infection was 32.0\%. There was a positive association of hookworm infection with living in single room $[A O R=2.8 ; 95 \% \mathrm{Cl} ; 1.32-5.81]$, living with domestic animals $[A O R=3.4 ; 95 \% \mathrm{Cl} ; 1.35-8.76]$, monthly income $\leq 1500$ Ethiopian birr $[\mathrm{AOR}=3.7 ; 95 \% \mathrm{Cl} ; 1.76-7.64]$, unavailability of latrine $[\mathrm{AOR}=2.2 ; 95 \% \mathrm{Cl}$; 1.03-4.55], habit of walking on barefoot $[\mathrm{AOR}=4.3 ; 95 \% \mathrm{Cl} ; 2.17-8.48]$ and not habit of hand washing before meal $[\mathrm{AOR}=3.4 ; 95 \mathrm{Cl} ; 1.14-10.12]$

*Correspondence: sewny2009@gmail.com

'Department of Medical Laboratory Technologist at Felege Hiote Specialized referral hospital, Bahir Dar, Ethiopia

Full list of author information is available at the end of the article

C C The Author(s). 2020 Open Access This article is licensed under a Creative Commons Attribution 4.0 International License, which permits use, sharing, adaptation, distribution and reproduction in any medium or format, as long as you give appropriate credit to the original author(s) and the source, provide a link to the Creative Commons licence, and indicate if changes were made. The images or other third party material in this article are included in the article's Creative Commons licence, unless indicated otherwise in a credit line to the material. If material is not included in the article's Creative Commons licence and your intended use is not permitted by statutory regulation or exceeds the permitted use, you will need to obtain permission directly from the copyright holder. To view a copy of this licence, visit http://creativecommons.org/licenses/by/4.0/. The Creative Commons Public Domain Dedication waiver (http://creativecommons.org/publicdomain/zero/1.0/) applies to the data made available in this article, unless otherwise stated in a credit line to the data. 


\begin{abstract}
(Continued from previous page)
Conclusion: This study showed high prevalence of hookworm infection among pregnant women in the study area. Living in single room, living with domestic animals, monthly income $\leq 1500$ Ethiopian birr, unavailability of latrine, habit of walking on barefoot and not habit of hand washing before meal had positive association with hookworm infection. Public health measure should focus on availability latrine and separation of humans and domestic animals room to decrease prevalence of hookworm infection among pregnant women.
\end{abstract}

Keywords: Hookworm, Prevalence, Pregnancy, Antenatal care, Dembecha District, Ethiopia

\section{Background}

Globally, 700 million people including 44 million pregnant women are infected with hookworm. It is also a cause of 3000 to 65,000 deaths annually [1]. In Sub-Saharan African, 37.7 million women of reproductive age are infected with it and those approximately 6.9 million are pregnant women [2]. In this region, countries with the highest prevalence of hookworm are Nigeria, Democratic Republic of Congo, Angola, Côte d'Ivoire and Ethiopia. It ranks at the top of major causes of disease burden because of the anemia that result from the blood feedings of the adult parasite [3]. The effect of hookworm infection on pregnant women and their fetus greatly varies from asymptomatic to sever infection that results in malnutrition, anemia, intrauterine growth retardation and spontaneous abortion [4]. It is also a leading cause of maternal and child illness in developing countries of the tropics and subtropics [3]. All pregnant women who were infected with hookworm were found to be anemic [5]. Anemia due to hookworm infection costs $\$ 20.9$ billion worldwide. It also resulted in $\$ 11.0$ billion in productivity losses in Africa [6]. Majority of infected individuals live in povertystricken areas with poor environmental sanitation [1]. The study done in southern region of Ethiopia showed that hookworm infection is the second most prevalent intestinal parasite among pregnant women with the mean prevalent of 7\% [7]. Even if there is current global control efforts based on periodic mass anthelmintic administration, it is unsustainable [8]. In Ethiopia, deworming is a component of antenatal care (ANC) but use of drugs to control intestinal parasites during pregnancy is least common among rural women, women with no education and women in households in the lowest and middle wealth quartile. There is limited information about hookworm infection in the study area. Thus, this study aimed at describing the prevalence of hookworm infection among pregnant women attending antenatal care in Dembecha district and determining factors associated with hookworm infection.

\section{Methods}

\section{Study area and period}

Institutional based cross sectional study was conducted from February1 to March 30, 2017 in Dembecha district,
Northwest Ethiopia. Dembecha district is bordered by Debre Elias district in the South, Jabitenan district in West, Dega Damot district in the North and Machakle district in the East. It is also located 350 and $217 \mathrm{~km}$ away from the capital city of Ethiopia (Addis Ababa) and capital city of Amhara Region (Bihar Dar), respectively. The sea level elevation of the district is $2083 \mathrm{~m}$. Humidity of the district is 54\%. The total population of the district was 156,665 and populations' density is 133.08 . The district has a latitude and longitude of $10^{\circ} 33^{\prime} \mathrm{N} 37^{\circ} 29^{\prime} \mathrm{E} /$ $10.550^{\circ} \mathrm{N} 37.483^{\circ} \mathrm{E}$ with an elevation of $2083 \mathrm{~m}$ above sea level. The district has six governmental health centers [9].

\section{Sample size and sampling technique}

The required sample size was computed by using single population proportion formula with the assumption of 95\% CI and 7\% prevalence rate of hookworm infection among pregnant women which was done in Hossana, Southern Ethiopia [7]. 10\% non- response rate and 3\% margin error used to obtain total sample size 306 . The total sample size was proportionally allocated for the six health centers based on the number of pregnant women who were registered in the health centers. Study participants in each health center were selected by systematic random sampling during the study period. Total number of pregnant women who expected to visit the health centers during data collection period was 881 . Sampling fraction $(\mathrm{K})$ was obtained by dividing the total pregnant women who were expected to visit the health centers for the total sample size. The first study participant was selected by lottery method and then every $\mathrm{K}$ interval (3rd) interval in each health center was included in the study.

\section{Data collection procedure}

Semi structured questionnaire was used to collect data using face to face interview technique by 6 trained laboratory technicians. The questionnaire had three sections that include socio-demographic information, personal, and environmental characteristics.

Questionnaire was prepared in English and translated into Amharic and translated back into English to check its consistency. The Amharic version questionnaire was used for data collection. Unique code was given for each questionnaire and laboratory request format. After 
asking the participants consent, they were interviewed and requested to give stool sample in clean dry cup. Similar codes were written on the cups with questionnaire and the laboratory request format. A fecal sample of all study participants was examined using wet mount preparation. A drop of fresh physiological saline was placed on a clean slide approximately $1 \mathrm{~g}$ of stool sample was added.

The preparation was covered with cover slip and examined under microscope for the presence or absence of hookworm ova.

\section{Data quality control}

Six diploma laboratory technicians were selected for data collection. And three laboratory professionals were selected for supervision. Training was given for both data collectors and supervisors for 2 days about the objective, process of data collection and standard operating procedure. Pretest was done on 16 pregnant women at Amanual health center in Machakle district before the actual data collection. Some clarifications and correction on the questionnaire was made after the pretest. Standard operating procedure (SOP) was used for laboratory procedures. Close supervision was under taken by supervisors during data collection. Specimens were selected randomly and re-examined by supervisors for cross checking the accuracy of laboratory results.

\section{Data analysis}

Collected data was coded and entered by using Epi data version 3.1 and then it was exported to SPSS version 20 for analysis. Descriptive, bivariate and multivariate analysis was done. After bivariate logistic regression analysis, all variables with a $p$-value less than 0.25 were entered into multivariate logistic regression to identify significant factors for the occurrence of hookworm infection among pregnant women. $P$-value less than 0.05 were used as cut off point for presence of statistical significance. Model goodness of fitness was checked by Hosmer and Lemeshow Test $(p$-value $=0.071)$. Tables and texts were used to present the result of the analyzed data.

\section{Results}

\section{Socio-demographic characteristics}

A total of 306 pregnant women were included in the study with $98.04 \%$ response rate. The mean age of the study participants was $26.56 \pm 5.94$ year. Among the total study participants, 290 (96.7\%), 176(58.7\%), 286 (95.3\%) and165 (55\%) were married, rural residence, Orthodox and farmers respectively. Half, 152 (50.7\%) of women could not able to read and write. Regarding to monthly income, 208 (69.3\%) of the study participants earned $>1500$ Ethiopian birr (Table 1).
Table 1 Socio-demographic and pregnancy related characteristics of pregnant women who attended antenatal care at governmental health centers in Dembecha district, North West Ethiopia, $2017(n=300)$

\begin{tabular}{|c|c|c|}
\hline Variable & Frequency & $\overline{\text { Percentage }}$ \\
\hline \multicolumn{3}{|l|}{ Residence } \\
\hline Urban & 124 & 41.3 \\
\hline Rural & 176 & 58.7 \\
\hline \multicolumn{3}{|l|}{ Age } \\
\hline$\leq 18$ year & 12 & 4.0 \\
\hline 19-24 year & 143 & 47.7 \\
\hline$\geq 25$ year & 145 & 48.3 \\
\hline \multicolumn{3}{|l|}{ Marital status } \\
\hline Married & 290 & 96.7 \\
\hline Single & 5 & 2.0 \\
\hline Divorced & 4 & 1.3 \\
\hline Widowed & 1 & 0.3 \\
\hline \multicolumn{3}{|l|}{ Educational status } \\
\hline Unable read and write & 152 & 50.5 \\
\hline Able to read and write but no formal education & 33 & 11 \\
\hline Primary & 70 & 23.3 \\
\hline Secondary & 36 & 12 \\
\hline Above secondary & 9 & 3 \\
\hline \multicolumn{3}{|l|}{ Religion } \\
\hline Orthodox & 286 & 95.3 \\
\hline Muslim & 10 & 3.3 \\
\hline Catholic & 1 & 0.3 \\
\hline Protestant & 3 & 1 \\
\hline \multicolumn{3}{|l|}{ Occupation } \\
\hline House wife & 36 & 12 \\
\hline Government employee & 18 & 6.0 \\
\hline Farmer & 165 & 55.0 \\
\hline Merchant & 73 & 24.3 \\
\hline Others $^{\mathrm{a}}$ & 8 & 2.7 \\
\hline \multicolumn{3}{|l|}{ Income } \\
\hline$>1500$ & 208 & 69.3 \\
\hline$\leq 1500$ & 92 & 30.7 \\
\hline \multicolumn{3}{|l|}{ First pregnancy } \\
\hline Yes & 127 & 42.3 \\
\hline No & 173 & 57.7 \\
\hline \multicolumn{3}{|c|}{ If not, number of pregnancy including the present one } \\
\hline 2 & 53 & 30.6 \\
\hline$\geq 3$ & 120 & 69.4 \\
\hline \multicolumn{3}{|l|}{ Month of current pregnancy } \\
\hline$\leq 3$ & 84 & 28 \\
\hline $4-6$ & 123 & 41 \\
\hline$\geq 7$ & 93 & 31 \\
\hline
\end{tabular}

Others $^{\mathrm{a}}$ = Daily labors, 6 (2\%) and Students, 2 (o.7\%). 
Table 2 Environmental and personal hygiene characteristics among pregnant women who attended at governmental health centers in Dembecha district, North West Ethiopia, 2017 ( $n=$ 300)

\begin{tabular}{lll}
\hline Variables & Frequency & Percentage \\
\hline Number of living rooms & & \\
1 & 89 & 29.7 \\
$\geq 2$ & 211 & 70.3
\end{tabular}

Availability of latrine

$\begin{array}{lll}\text { Yes } & 162 & 54.0 \\ \text { No } & 138 & 46.0\end{array}$

types of latrine $(n=162)$

Improved

Traditional

Use of the latrine $(n=162)$

Always

Some times

52

Place of defecation $(n=138)$

Open field 118

From neighbor toilet

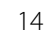

Others

Open defecation seen

Yes 159

No

141

Location of open defecation seen $(n=159)$

Near the river

In the main road

Through the bush

Other

Domestic animal presence

Yes

No

98

Type of animals

Cat

Dog

Cow/ox

Goat

Sheep

Others

Source of drinking water

Pipe

River

Well

Spring

Habit of hand washing before meal
Table 2 Environmental and personal hygiene characteristics among pregnant women who attended at governmental health centers in Dembecha district, North West Ethiopia, 2017 ( $n=$ 300) (Continued)

\begin{tabular}{|c|c|c|}
\hline Variables & Frequency & Percentage \\
\hline \multicolumn{3}{|c|}{ Have you a habit taking of bath } \\
\hline Yes & 219 & 73 \\
\hline No & 81 & 27 \\
\hline \multicolumn{3}{|c|}{ How often do you take $(n=219)$} \\
\hline$>1$ a week & 43 & 19.6 \\
\hline Once a week & 74 & 33.8 \\
\hline Once a month & 99 & 45.2 \\
\hline Others & 3 & 1.4 \\
\hline \multicolumn{3}{|c|}{ Where do you take $(n=219)$} \\
\hline Inside the house & 97 & 44.3 \\
\hline Outside the house & 122 & 55.7 \\
\hline \multicolumn{3}{|c|}{ Place of bath $(n=122)$} \\
\hline Pond & 61 & 50 \\
\hline At water fall & 48 & 39.3 \\
\hline${ }^{\text {aOOthers }}$ & 13 & 10.7 \\
\hline \multicolumn{3}{|c|}{ Habit of raw fruit eating } \\
\hline Yes & 208 & 69.3 \\
\hline No & 92 & 30.7 \\
\hline \multicolumn{3}{|c|}{ Washing fruit before eating $(n=208)$} \\
\hline Yes & 83 & 39.9 \\
\hline No & 125 & 60.1 \\
\hline \multicolumn{3}{|c|}{ Habit of shoe wearing } \\
\hline Yes & 162 & 54 \\
\hline No & 138 & 46 \\
\hline
\end{tabular}

Others $^{\mathrm{a}}=$ under the tree, 5 (4.1\%), Latrine, 4 (3.3\%) and Backyard, 4 (3.3\%).

Environmental and personal hygiene characteristics Nearly three-fourth, 211(70.3\%) of the respondents were living in house having two or more rooms. More than half, $162(54 \%)$ of the participants were living in households that have toilet facility but majority, 111 (68.5) of participants' toilets were made in a traditional way. Among participants, 138 (46\%) were living in the households without toilet facility, and most $118(85.6 \%)$ of them had habit of open defecation. Majority, 202(67.3\%) of the respondents have lived with domestic animals. Two hundred nineteen $(73 \%)$ of participants had habit of taking bath. Most 208(69.3\%) of the respondents had the habit of eating raw fruit or vegetable. Less than half $138(40 \%)$ of the participants had habit of walking on barefoot (Table 2).

Prevalence of hookworm infection

Based on stool microscopic result, 96 (32\%) 95\% CI (26.3-37\%) of the respondent had hookworm infection. 
Table 3 Multivariate logistic regression analysis of factors associated with hookworm infection among pregnant women who attended antenatal care at governmental health centers in Dembecha district, North West Ethiopia, $2017(n=300)$

\begin{tabular}{|c|c|c|c|c|c|}
\hline \multirow[t]{2}{*}{ Characteristics } & \multicolumn{2}{|c|}{ Hookworm infection } & \multirow[t]{2}{*}{ COR $((95 \% \mathrm{Cl})$} & \multirow[t]{2}{*}{ AOR (95\% Cl) } & \multirow{2}{*}{$\begin{array}{l}\boldsymbol{P} \text { - } \\
\text { value }\end{array}$} \\
\hline & $\overline{\mathrm{No}}$ & Yes & & & \\
\hline \multicolumn{6}{|l|}{ Income } \\
\hline$>1500$ & 160 & 48 & 1 & 1 & \\
\hline$\leq 1500$ & 44 & 48 & $3.6(2.16-6.12)$ & $3.7(1.76-7.64)$ & $0.001^{*}$ \\
\hline \multicolumn{6}{|c|}{ Number of living room } \\
\hline$\geq 2$ & 155 & 56 & 1 & 1 & \\
\hline 1 & 49 & 40 & $2.3(1.34-3.79)$ & $2.8(1.32-5.80)$ & $0.007^{*}$ \\
\hline \multicolumn{6}{|c|}{ Availability of latrine } \\
\hline Yes & 132 & 30 & 1 & 1 & \\
\hline No & 72 & 66 & $4.0(2.40-6.77)$ & $2.2(1.03-4.55)$ & $0.042^{*}$ \\
\hline \multicolumn{6}{|c|}{ Living with domestic animal } \\
\hline No & 82 & 16 & 1 & 1 & \\
\hline Yes & 122 & 80 & $3.4(1.84-6.17)$ & $3.4(1.35-8.76)$ & $0.009^{*}$ \\
\hline \multicolumn{6}{|c|}{ Habit of walking barefoot } \\
\hline Yes & 140 & 22 & 1 & 1 & \\
\hline No & 64 & 74 & $7.4(4.20-12.88)$ & $4.3(2.17-8.47)$ & $0.000^{*}$ \\
\hline \multicolumn{6}{|c|}{ Not habit of hand washing before meal } \\
\hline Yes & 178 & 64 & 1 & 1 & \\
\hline No & 26 & 32 & $3.4(1.89-6.18)$ & $3.4(1.14-10.11)$ & $0.028^{*}$ \\
\hline \multicolumn{6}{|l|}{ Residence } \\
\hline Urban & 97 & 26 & 1 & 1 & \\
\hline Rural & 107 & 70 & $2.2(1.28-3.62)$ & $0.3(0.05-2.48)$ & 0.290 \\
\hline \multicolumn{6}{|l|}{ Age in years } \\
\hline $15-18$ & 10 & 10 & 1 & 1 & 0.342 \\
\hline $19-25$ & 103 & 103 & $1.9(0.40-9.25)$ & $1.6(0.20-12.88)$ & 0.650 \\
\hline$>25$ & 91 & 91 & $2.9(0.62-14.05)$ & $2.9(0.32-25.60)$ & 0.347 \\
\hline \multicolumn{6}{|l|}{ First pregnancy } \\
\hline Yes & 95 & 32 & 1 & 1 & \\
\hline No & 109 & 64 & $1.7(1.05-2.89)$ & $2.1(0.91-4.96)$ & 0.080 \\
\hline \multicolumn{6}{|c|}{ Month of current pregnancy } \\
\hline$\leq 3$ & 65 & 19 & 1 & 1 & 0.442 \\
\hline $4-6$ & 79 & 44 & $1.90(1.01-3.57)$ & $1.4(0.57-3.26)$ & 0.480 \\
\hline$\geq 7$ & 60 & 33 & $1.9(0.97-3.66)$ & $0.8(0.33-2.22)$ & 0.738 \\
\hline \multicolumn{6}{|c|}{ Have seen open defecation } \\
\hline No & 111 & 30 & 1 & 1 & \\
\hline Yes & 93 & 66 & $2.6(1.57-4.38)$ & $1.2(0.52-2.68)$ & 0.687 \\
\hline \multicolumn{6}{|c|}{ Habit of eating raw fruit } \\
\hline No & 63 & 18 & 1 & 1 & \\
\hline Yes & 141 & 78 & $1.7(1.00-3.06)$ & $1.9(0.94-4.04)$ & 0.071 \\
\hline \multicolumn{6}{|c|}{ Habit of bathing } \\
\hline Yes & 162 & 57 & 1 & 1 & \\
\hline No & 42 & 39 & $2.6(1.55-4.48)$ & $0.7(0.25-1.86)$ & 0.452 \\
\hline
\end{tabular}

Drinking water source 
Table 3 Multivariate logistic regression analysis of factors associated with hookworm infection among pregnant women who attended antenatal care at governmental health centers in Dembecha district, North West Ethiopia, 2017 ( $n=300$ ) (Continued)

\begin{tabular}{|c|c|c|c|c|c|}
\hline \multirow[t]{2}{*}{ Characteristics } & \multicolumn{2}{|c|}{ Hookworm infection } & \multirow[t]{2}{*}{ COR ((95\% Cl) } & \multirow[t]{2}{*}{ AOR (95\% Cl) } & \multirow{2}{*}{$\begin{array}{l}\boldsymbol{P}_{-} \\
\text {value }\end{array}$} \\
\hline & No & Yes & & & \\
\hline Pipe & 86 & 25 & 1 & 1 & 0.155 \\
\hline River & 6 & 14 & $8.0(2.79-23.05)$ & $1.7(0.25-11.10)$ & 0.590 \\
\hline Well & 69 & 36 & $1.8(0.98-3.27)$ & $0.5(0.12-2.41)$ & 0.407 \\
\hline Spring & 43 & 21 & $8.0(2.79-23.05)$ & $0.4(0.07-1.94)$ & 0.234 \\
\hline
\end{tabular}

*significant with $p<0.05$

\section{Factors associated with hookworm infection}

In Multivariable analysis; monthly income $(\mathrm{AOR}=3.7$; 95\% CI; 1.76-7.64), living with a single room (AOR =2.8; $95 \% \mathrm{CI} ; 1.32-5.81)$, unavailability of latrine $(\mathrm{AOR}=2.2$; 95\% CI; 1.03-4.55), living with domestic animals (AOR = 3.4; 95\% CI; 1.35-8.76), habit of walking on barefoot $(\mathrm{AOR}=4.3 ; 95 \% \mathrm{CI} ; 2.17-8.48)$ and not habit of hand washing before meal $(\mathrm{AOR}=3.4 ; 95 \mathrm{CI} ; 1.14-10.12)$ were significantly associated with hookworm infection (Table 3).

\section{Discussion}

This study showed that the prevalence of hookworm infection was $32.0 \%$. This finding is lower than the study conducted among pregnant women in Vietnam 78.15\% [10], Andhra Pradesh 78.14\% [11], Ghana, 45\% [12] and Uganda 45\% [13]. This low prevalence in this study might be geographic difference and time gap where those studies were conducted averagely 5 years ago. The finding of this study almost similar with the study conducted in Nigeria $35.8 \%$ [4]. The finding of this study had also higher prevalence of hookworm infection than the study done in Hossana, Ethiopia 7.0\% [7], Addis Ababa, Ethiopia 1.3\% [14] and Bahir dare, Ethiopia 5.5\% [15]. This higher prevalence in this study might be due to socio demographic difference. In the previous studies the majority of participants were in urban dweller but in this study majority of participants were rural dweller. Pregnant women who lived in a single room were 2.8 times more likely to be infected by hookworm than pregnant women who lived in $\geq 2$ living rooms. This finding is consistent to the study conducted in Southern Thailand [16]. This might be due to poverty stricken households with poor sanitation [1]. In this study, pregnant women whose monthly income less than or equal to 1500 Ethiopian birr were 3.7 times more likely to be infected by hookworm than pregnant women whose monthly family income greater than 1500 Ethiopian birr. This study is almost similar with the study done in Hossanna, Ethiopia [7]. This might to be due to not affording to buy shoes and might led to walking on barefoot which predisposes for hookworm infection [17]. Pregnant women who lived in household that have not toilet facility had almost 2.2 times more likely to be infected by hookworm than pregnant women who lived in households that have toilet facility. This finding is almost similar with the study conducted in southern Thailand [14]. This might be due to open defecation that lead contamination with faces may cause hookworm infection [18]. In this study, pregnant women with habit of barefoot had almost 4.3 times more likely to be infected by hookworm than those who did not the habit. This study is almost comparable with the study done in Thailand [16, 19], Dhare, India [20] and Hossana, Ethiopia [7]. Walking on barefoot might predispose to hookworm infection whose infective stage is found in soil $[8,17,18,21,22]$. The result of the study showed that pregnant women who had not habit of hand washing were 3.4 times more likely to be infected by hookworm than those who had habit of hand washing before meal. This study is almost similar with the study conducted in Peninsular Malaysia [23]. This might be due to contamination of hand with soil and faces which leads hookworm transmission through the ingestion of larvae [17].

In this study, pregnant women who lived with domestic animals had almost 3.4 times more likely infected by hookworm than those who did not live with domestic animals. This result is almost similar with the study conducted in Malaysia [23]. This might to be due to domestic animals may contaminate with human faces which contain hookworm larvae. It can lead a hookworm infection by touching contaminated animal [18]. The limitations of the study are less sensitive with compared to concentration diagnostic technique to detect light hookworm infection and the study also did not show the effect and the load of hookworm infection among pregnant women.

\section{Conclusion}

This study showed that high prevalence of hookworm infection among pregnant women in the study area. Infections with hookworm among pregnant women were positively associated with not habit of hand washing 
before meal, living with a single room, monthly income, unavailability of latrine, living with domestic animals and habit of walking on barefoot. Therefore, Public health measures should contain to emphasis the importance of environmental, personal hygiene and preventive chemotherapy (deworming) is recommended as a public health intervention for all pregnant women in the study area as the base line prevalence of hookworm infections is high.

\section{Supplementary information}

Supplementary information accompanies this paper at https://doi.org/10 1186/s12884-020-03134-0.

Additional file 1. Questionnaire (Amharic and English language version)

\section{Abbreviations}

ANC: Antenatal care; AOR: Adjusted odds ratio; Cl: Confidence interval; COR: Crude odds ratio; SPSS: Statistical package for social science

\section{Acknowledgements}

We would like to acknowledge Debre Markos University, College of Health Sciences and Department of Public health. We would also like to acknowledge data collectors and supervisors for collecting appropriate data, and all pregnant women who were willingly took part in this study.

\section{Authors' contributions}

SW wrote the proposal, involved in study design, analyzed the data, drafted paper. MW approved the design and the proposal. MK involved in data entry, revised subsequent drafts of the paper and reviewing of the manuscript. SA: revised manuscript. All authors read and approved the final manuscript.

\section{Funding}

This work was funded by ourselves

\section{Availability of data and materials}

The data sets generated during the current study are available from corresponding author on reasonable request.

\section{Ethics approval and consent to participate}

Ethical clearance was obtained from Debre Markos University Institutional Review Board committee. Permission letter was sought from Dembecha district health office. Informed written consent was obtained from each participant. Written consent was received from adult next-of-kin/parental when the respondent was less than 16 years of age and verbal assent was obtained from less than 16 years age of participants. The study participants had the right to refuse in the study or withdraw at any time during the interview was respected. The information obtained from the study participant was maintained its confidentiality by not writing name of the study participants on the questionnaire paper.

\section{Consent for publication}

Not applicable

\section{Competing interests}

The authors declare that they have no competing interests

\section{Author details}

'Department of Medical Laboratory Technologist at Felege Hiote Specialized referral hospital, Bahir Dar, Ethiopia. ${ }^{2}$ Department of Public Health, College of Health Sciences, Debre Markos University, Debre Markos, Ethiopia. ${ }^{3}$ Resident of Field Epidemiology, Department of Epidemiology and Biostatistics, College of Medicine and Health Sciences, Bahir Dar University, Bahir Dar, Ethiopia. ${ }^{4}$ Department of Midwifery, College of Medicine and School of Health Sciences, Bahir Dar, University, Bahir Dar, Ethiopia.
Received: 4 February 2019 Accepted: 26 July 2020

Published online: 12 August 2020

\section{References}

1. Walana W, Nana E, Crowther S. Prevalence of hookworm infection: a retrospective study in Kumasi. Asian Pac J Trop Biomed. 2014;4(1):158-61.

2. Brooker S, Hotez PJ, Bundy DAP. Hookworm-related Anaemia among pregnant women: a systematic review. PLoS Negl Trop Dis. 2008;2(9):1-9.

3. Peter J. The development impact of the neglected tropical diseases (NTDs). Expert Paper. 2011:6-13.

4. Alli JA, Okonko IO, Kolade AF, Nwanze JC, Dada VK, Ogundele M. Prevalence of intestinal nematode infection among pregnant women attending antenatal clinic at the university college hospital, Ibadan, Nigeria. Pelagia Res Library. 2011;2(4):4-5.

5. Baido SE, Tay SCK, Obiri-Danso K, Abruquah HH. Intestinal helminth infection and anaemia during pregnancy: a community based study in Ghana. J Bacteriol Res. 2010;2(2):11-3.

6. Bartsch SM, Hotez PJ, Asti L, Zapf KM, Bottazzi ME, Diemert DJ, et al. The global economic and health burden of human hookworm infection. PLoS Negl Trop Dis. 2016;10(9):e0004922. https://doi.org/10.1371/journal.pntd. 0004922.

7. Tesfaye D.J., Beshir W.G., Dejene T. and Tewelde, T. Prevalence of intestinal helminthiases and associated factors among pregnant women attending antenatal clinic of Nigist Eleni Mohammed Memorial Hospital, Hossana, Southern Ethiopia. Open Access Library J; 2015, 2: e1660. http://dx.doi.org/ https://doi.org/10.4236/oalib.1101660.

8. Plotkin S, Diemert DJ, Bethony JM, Hotez PJ. Hookworm vaccines. Oxf J Med Health Clin Infect Dis. 2007:46(2):282-8.

9. Ethiopian fiscal year 2009 Dembecha health office annual plan, 2008, Dembecha.

10. Sant R. P., Sonia R. C., Tran Q. P., Gerard J. C., Damien J., Sally K., Nong T. T. ,Lachlan M.G., Antonio M. and Beverley A .B. Anemia, Iron deficiency, meat consumption, and hookworm infection in women of reproductive age in Northwest Vietnam. Am Soc Trop Med Hyg; 2008, 78(3): 375-381.

11. Kudaravalli J, Madhavi S, Nagaveni D, Deshpande N, Rama M. Anemia, Iron deficiency, meat consumption, and hookworm infection in women of reproductive age in rural area in Andhra Pradesh. Ann Biol Res. 2011;2(3): 209-16

12. Humphries D, Mosites E, Otchere J, Twum WA, Woo L, Jones-Sanpei $H_{\text {, }}$ Harrison LM, Bungiro RD, Benham-Pyle B, Bimi L, Edoh D. Epidemiology of hookworm infection in Kintampo North Municipality, Ghana: patterns of malaria coinfection, anemia, and albendazole treatment failure. Am Soc Trop Med Hyg. 2011;84(5):792-800.

13. Mpairwe H, Ndibazza J, Webb EL, Nampijja M, Muhangi L, Apule B, Lule S, Akurut $H$, Kizito D, Kakande M, Jones FM, Fitzsimmons CM, Muwanga M, Rodrigues LC, Dunne DW, Elliott AM. Maternal hookworm modifies risk factors for childhood eczema: results from a birth cohort in Uganda. Pediatr Allergy Immunol. 2014:25:481-8.

14. Intestinal GW. Parasitic infection in pregnant women attending antenatal care at Gandhi Memorial Hospital, Addis Ababa, Ethiopia. Harar Bull Health Sci. 2015;5:85

15. Derso A, Nibret $E$, Munshea A. Prevalence of intestinal parasitic infections and associated risk factors among pregnant women attending antenatal care center at Felege Hiwot referral hospital, Northwest Ethiopia. BMC Infect Dis. 2016;16(530):3-5.

16. Liabsuetrakul T. Epidemiology and the effect of treatment of soiltransmitted helminthiasis in pregnant women in southern Thailand Southeast Asian J Trop Med Public Health. 2009;40(2):211-8.

17. Hotez PJ, Bethony J, Bottazzi ME, Brooker S, Diemert D, Loukas A. New technologies for the control of human hookworm infection. Trends in parasitology. 2006;22(7):327-31.

18. Fiester RF. Division of Infectious Disease Department of Medicine University of Illinois Hospital PO Box 6990 Chicago, 111. 60616. Science. 1969;166: $1032-4$

19. Jiraanankul V, Aphijirawat W, Mungthin M, Khositnithikul R, Rangsin R, Traub RJ, Piyaraj P, Naaglor T, Taamasri P, Leelayoova S. Incidence and risk factors of hookworm infection in a rural Community of Central Thailand. Am Soc Trop Med Hyg. 2011;84(4):594-8.

20. Prajapati R, Bhagore J. Study of intestinal parasite of adjacent villages of Dhar. Int J Sci Res (IJSR). 2015;4(5):941-3. 
21. Hotez PJ, Bethony J, Bottazzi ME, Brooker S, Buss P. Hookworm: "The great infection of mankind.". PLoS Med. 2005;2(3):e67.

22. Fenwick A. "The global burden of neglected tropical diseases". Public Health; 2012, 126 (3): 233-236.Doi:10.1016/j. puhe. 2011.11.015. PMID 22325616

23. Ngui R, Aziz S, Chua KH, Aidil RM, Lee SC, Tan TK, Sani MM, Arine AF, Rohela M, Lim A. Patterns and Risk Factors of Soil-Transmitted Helminthiasis among Orang Asli Subgroups in Peninsular Malaysia. Am Soc Trop Med Hyg. 2015; 93(2):361-70.

\section{Publisher's Note}

Springer Nature remains neutral with regard to jurisdictional claims in published maps and institutional affiliations.

Ready to submit your research? Choose BMC and benefit from:

- fast, convenient online submission

- thorough peer review by experienced researchers in your field

- rapid publication on acceptance

- support for research data, including large and complex data types

- gold Open Access which fosters wider collaboration and increased citations

- maximum visibility for your research: over $100 \mathrm{M}$ website views per year

At $\mathrm{BMC}$, research is always in progress.

Learn more biomedcentral.com/submissions 